\title{
Time-Domain Finite-Element Eddy-Current Homogenization of Windings using Foster Networks and Recursive Convolution
}

\author{
C. A. Valdivieso ${ }^{1,2,4}$, G. Meunier ${ }^{1}$, B. Ramdane ${ }^{1}$, J. Gyselinck ${ }^{3}$, C. Guerin ${ }^{4}$, and R. V. Sabariego ${ }^{2}$ \\ ${ }^{1}$ Univ. Grenoble Alpes, CNRS, Grenoble INP, G2Elab, F-38000 Grenoble, France \\ ${ }^{2}$ KU Leuven, Dept. Electrical Engineering, Campus EnergyVille, 3600 Genk, Belgium \\ ${ }^{3}$ Université Libre de Bruxelles, BEAMS department, 1050 Brussels, Belgium \\ ${ }^{4}$ Altair Engineering France, 38240 Meylan, France
}

\begin{abstract}
In this paper, we propose an alternative time-domain approach for the homogenization of multiturn windings with frequencydependent parameters in finite-element (FE) models. First, an elementary 2-D FE characterization of the conductors is carried out over the frequency range of interest to obtain the frequency-dependent parameters and account for the eddy-current effects in the winding. These complex coefficients are subsequently associated to an equivalent Foster network and translated into the time domain via the inverse Laplace transform. The resulting time-domain equations are solved with a recursive convolution scheme. No extra degrees of freedom are required in the homogenized winding. The results of the homogenized model present an excellent agreement with those obtained by an accurate but expensive FE model in which all turns are explicitly discretized. The proposed approach is further compared to a previously developed time-domain homogenization technique based on RL Cauer networks.
\end{abstract}

Index Terms-Eddy currents, finite element method, homogenization, proximity effect, skin effect, time domain, windings.

\section{INTRODUCTION}

$\mathbf{M}$ ULTITURN windings in electromagnetic devices may exhibit considerable skin and proximity effects when operated at sufficiently high frequencies. Therefore, a correct prediction of these effects constitutes a major design aspect for e.g., switched mode power supply (SMPS) transformers. The finite element (FE) method can directly include these effects by explicitly discretizing each winding turn. For most real-life applications, with possibly complex geometries, such approach leads to extremely fine meshes and a prohibitive number of unknowns. Even though some modeling techniques allow for efficient meshing by inserting preprocessed values in the boundary of the conductors [1], [2], [3], [4], [5]; the FE mesh still yields a considerable number of elements, especially in 3-D. Thus, the use of dedicated homogenization techniques is an excellent and indispensable alternative.

The simplest homogenized model for windings, the stranded model, considers a uniform current density in the winding window and ignores eddy-current effects at the FE resolution stage [6]. In the frequency domain, such effects can be added by means of complex frequency-dependent parameters: reluctivity and impedance, which may be obtained analytically [7], semianalytically [8] or by using an elementary FE model [9], [10], [11], [12]. Thereon, a time-domain extension, proposed in [13], [14], associates the frequency-dependent coefficients to RL Cauer networks and translates them to the time domain as real constants linked to differential equations, thanks to the inclusion of additional unknowns (flux densities in the homogenized winding and currents in the supply circuit).

In this paper, an alternative time-domain homogenization method is proposed. It uses the elementary FE model to

Manuscript received XXXX X, 20XX; revised XXX X, 20XX. Corresponding author: C. A. Valdivieso (email: cvaldivieso@altair.com). obtain the complex frequency-dependent parameters and then associates them to equivalent Foster networks by using the Vector-Fitting technique [15]. The inverse Laplace transform is subsequently applied to translate these parameters into the time domain. The resulting time-domain equations are solved with a recursive convolution (RC) scheme [16], which is a common technique in transmission line modeling [17], [18], [19], [20], [21]. The proposed method delivers highly accurate results and does not require additional unknowns in the homogenized winding. By way of validation, a 2-D 256-turn inductor is considered, for which a fine FE model provides an accurate reference solution. Furthermore, the proposed approach is compared to the use of an RL Cauer network as in [13], [14].

\section{Frequency-Domain Homogenization of WINDINGS WITH EDDY-CURRENT EFFECTS}

\section{A. Magnetodynamic Formulation}

A bounded domain $\Omega$ of the 2- or 3-D Euclidean space is considered. The conducting and nonconducting parts are denoted $\Omega_{c}$ and $\Omega_{n c}$, respectively, with $\Omega=\Omega_{c} \cup \Omega_{n c}$. Moreover, $\Omega_{c}$ is divided into a solid-conductor region $\Omega_{s}$ and a winding region $\Omega_{w}$ (subscripts $s$ and $w$, respectively), so that $\Omega_{c}=\Omega_{s} \cup \Omega_{w}$. The classical stranded model [6], used in $\Omega_{w}$, considers a uniform current density; while the solid conductor, used in $\Omega_{s}$, considers a nonuniform current density due to skin and proximity effects [22]. This way, in terms of the magnetic vector potential (MVP), the first-order ordinary differential equations (ODEs) arising from a FE discretization, containing the $N\left(N_{w}\right.$ in $\left.\Omega_{w}\right)$ shape and test functions, read:

$$
\begin{gathered}
{[M][A]+\left[D_{s}\right] \partial_{t}[A]=R_{s}^{-1}\left[C_{s}\right] V_{s}+\left[C_{w}\right] I_{w}} \\
V_{s}=\left[C_{s}\right]^{\top} \partial_{t}[A]+R_{s} I_{s} \\
V_{w}=\left[C_{w}\right]^{\top} \partial_{t}[A]+R_{w} I_{w}
\end{gathered}
$$


where $[M] \in \mathbb{R}^{N \times N}$ is the reluctivity-dependent stiffness matrix, $\left[D_{s}\right] \in \mathbb{R}^{N \times N}$ the conductivity-dependent eddy-current matrix, $\left[C_{s}\right] \in \mathbb{R}^{N \times 1}$ and $\left[C_{w}\right] \in \mathbb{R}^{N \times 1}$ the connectivity matrices, $[A] \in \mathbb{R}^{N \times 1}$ the MVP unknown vector; $V_{s}, V_{w}$, $I_{s}, I_{w}, R_{s}$ and $R_{w}$ the respective voltages, currents and resistances, and the ${ }^{T}$ denotes a transposition.

Thereupon, in the frequency domain, the winding eddycurrent effects can be included by replacing the reluctivity $\nu$ (in $\Omega_{w}$ ) and $R_{w}$ by an equivalent complex frequencydependent reluctivity $\boldsymbol{\nu}_{e}$ and an equivalent impedance $\boldsymbol{Z}_{e}$, respectively [7], [10], [11]. In the following, the obtention of these complex frequency-dependent parameters is explained.

\section{B. Characterization of Multiturn Windings}

\section{1) Considerations}

A winding of $N_{c}$ periodically spaced turns carrying the same net current $\boldsymbol{I}_{w}$ is considered, since parasitic capacitive effects are disregarded. The turns have conductivity $\sigma$ (or, resistivity, $\rho=1 / \sigma$ ) and a reluctivity $\nu=\nu_{0}$ (or, permeability, $\left.\mu=\mu_{0}=1 / \nu_{0}\right)$. The skin depth at frequency $f$, or pulsation $\omega=2 \pi f$, is given by $\delta_{s}=\sqrt{\nu / \pi f \sigma}$. The insulation between the conductors is nonmagnetic $\left(\nu=\nu_{0}\right)$ and the fill factor is denoted by $\lambda$. Underlined symbols indicate vectors and, for frequency-domain calculations, the complex notation (symbols in bold, $\boldsymbol{\imath}$ the imaginary unit and the ${ }^{*}$ indicates a conjugate value) is adopted.

On a macroscopic scale, periodic structures can be homogenized in terms of equivalent constitutive parameters. Windings are by nature periodic structures (bundles of wires) and their eddy-current effects may be accurately represented by means of an equivalent impedance $Z_{e}$, accounting for the skin effect, and an equivalent reluctivity $\boldsymbol{\nu}_{e}$, accounting for the proximity effect [10], [11].

\section{2) Elementary 2-D FE model}

The complete eddy-current characterization of a winding can be carried out by means of an elementary 2-D FE model (in Cartesian coordiantes: $x, y, z$ ), which consists of a cell comprising the conductor (modeled as a solid conductor) and the insulation around it. In Fig. 1 such a FE model is shown for one of the conductors to be used in the application: a round conductor with square packing (radius $r=1.15 \mathrm{~mm}$, $\sigma=59 \mathrm{MS} / \mathrm{m}$ and $\lambda=0.43)$. The horizontal and vertical lengths of the elementary cell are denoted $l_{x}$ and $l_{y}$, respectively, and its section $\mathcal{A}_{c}$ is given by $l_{x} l_{y}$. The complex power $\boldsymbol{S}$ (in VA) absorbed by the elemantary cell is calculated from the local flux density $\boldsymbol{b}$ and the local current density $\boldsymbol{j}$ as

$$
\boldsymbol{S}=P+\boldsymbol{\imath} Q=\frac{l_{z}}{2} \int_{\Omega_{c e l l}}\left(\frac{\underline{\boldsymbol{j}}^{*}}{\sigma}+\boldsymbol{\imath} \omega \nu_{0} \underline{\boldsymbol{b}}^{*}\right) d \Omega_{c e l l},
$$

where $P$ is the active power, $Q$ the reactive power and $l_{z}$ the length along the third dimension.

If a winding is described by the spatial reproduction of the elementary cell, periodic conditions are perfectly guaranteed and the eddy-current effects can be included by imposing either the net circulating current $\boldsymbol{I}_{s}$ or the average flux density $\boldsymbol{b}_{a v}$ on the cell. Hence, the skin- and proximity-effect

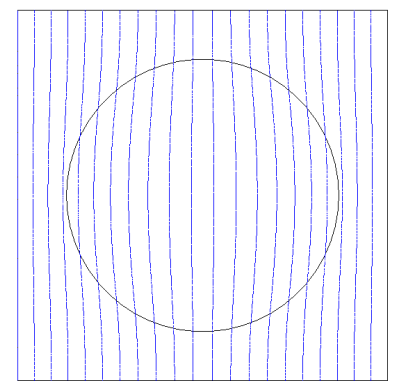

(a)

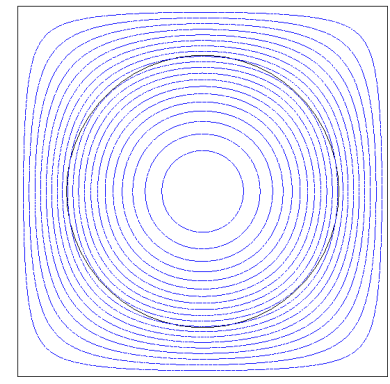

(c)

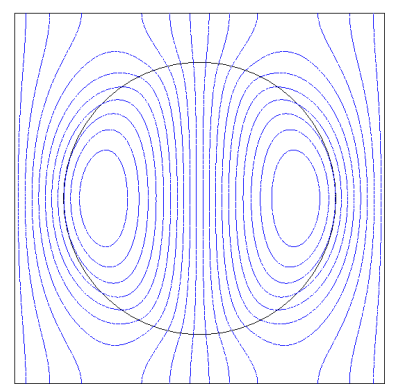

(b)

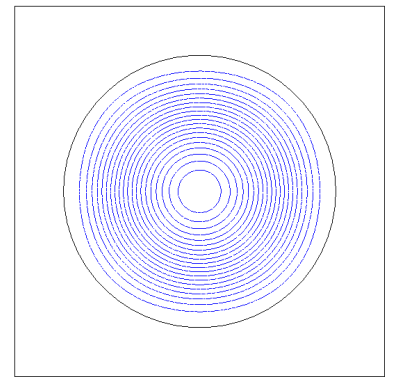

(d)
Fig. 1. Flux lines in the elementary cell at $20 \mathrm{kHz}$ : proximity effect (a) real and (b) imaginary parts and skin effect (c) real and (d) imaginary parts. Images obtained with Altair Flux ${ }^{\mathrm{TM}}$ [23].

characterization detailed hereafter is based on the redefinition of (4) in terms of $\boldsymbol{I}_{s}$ and $\boldsymbol{b}_{a v}$ as

$$
\boldsymbol{S}=\frac{1}{2}\left(\boldsymbol{Z}_{e} \boldsymbol{I}_{s} \boldsymbol{I}_{s}^{*}+\boldsymbol{\imath} \omega l_{z} \mathcal{A}_{c} \boldsymbol{\nu}_{e}^{*} \boldsymbol{b}_{a v} \boldsymbol{b}_{a v}^{*}\right)
$$

The equivalent impedance and the equivalent reluctivity can be decomposed into their real and imaginary parts: $\boldsymbol{Z}_{e}=R_{e}+$ $\boldsymbol{\imath} X_{e}$ and $\nu_{e}=\nu_{e}^{r}+\boldsymbol{\imath} \nu_{e}^{i}$, where $R_{e}$ is the equivalent resistance, $X_{e}$ the equivalent reactance, $\nu_{e}^{r}$ and $\nu_{e}^{i}$ the real and imaginary parts of the equivalent reluctivity.

\section{3) Proximity Effect}

Following the approach in [10] or [12], a pure proximityeffect excitation is obtained by imposing e.g. a vertical flux and a zero net circulating current $\left(\boldsymbol{I}_{s}=0\right)$. This is achieved with $\boldsymbol{a}_{z}=-1$ and $\boldsymbol{a}_{z}=1$ on the left and right boundaries, respectively, and Neumann conditions $\left(\partial \boldsymbol{a}_{z} / \partial n=0\right)$ on the upper and lower boundaries. The flux lines are shown in Figs. $1 \mathrm{a}$ and $1 \mathrm{~b}$. Via the complex power $S$ (5), the frequencydependent equivalent reluctivity $\nu_{e}$ is defined:

$$
\boldsymbol{\nu}_{e}(\omega)=2 \frac{Q+\boldsymbol{\imath} P}{\omega l_{z} \mathcal{A}_{c} \boldsymbol{b}_{a v} \boldsymbol{b}_{a v}^{*}} .
$$

Since the treated cell is composed of a round conductor with square packing, $\nu_{e}$ is a scalar quantity; however, for cells of arbitrary cross-section, $\boldsymbol{\nu}_{e}$ may become a tensor. Fig. 2 shows the frequency dependence up to $100 \mathrm{kHz}$ for the equivalent reluctivity $\left(\nu_{e}^{r}\right.$ and $\left.\nu_{e}^{i} \mathrm{FE}\right)$ obtained with the elementary FE model depicted in Fig. 1 .

4) Skin Effect

A pure skin-effect is obtained by imposing a sinusoidal current with a zero average flux density $\left(\boldsymbol{b}_{a v}=0\right)$. This is 


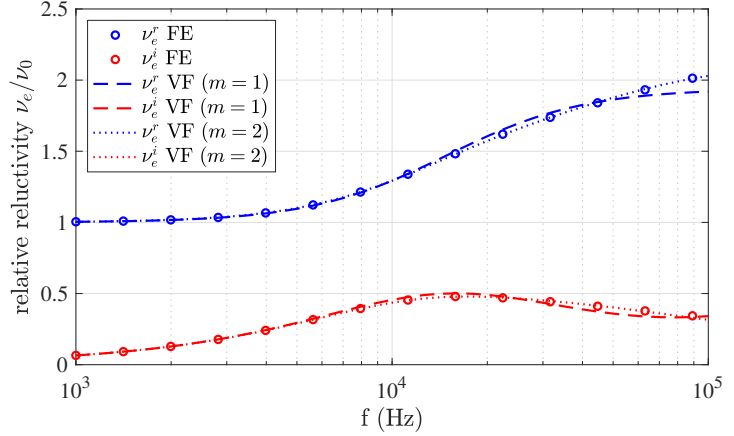

Fig. 2. Equivalent relative reluctivity (real and imaginary parts) versus frequency obtained with the elementary FE model and approximated with Vector Fitting VF

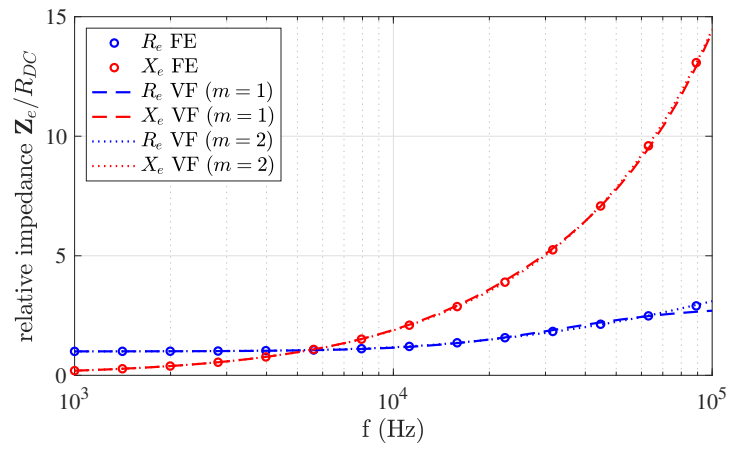

Fig. 3. Equivalent relative impedance (real and imaginary parts) versus frequency obtained with the elementary FE model and approximated with Vector Fitting VF.

achieved e.g. with $\boldsymbol{I}_{s}=1$ and $\boldsymbol{a}_{z}=0$ on the complete boundary of the elementary cell. The flux lines are shown in Fig. 1c and 1d Analogously, via the complex power $S$ (5), the equivalent winding impedance $\boldsymbol{Z}_{e}$ is defined:

$$
\boldsymbol{Z}_{e}(\omega)=2 N_{c} \frac{P+\imath Q}{\boldsymbol{I}_{s} \boldsymbol{I}_{s}^{*}} .
$$

Fig. 2 shows the frequency dependence up to $100 \mathrm{kHz}$ for the equivalent impedance $\left(R_{e}\right.$ and $\left.X_{e} \mathrm{FE}\right)$ obtained with the elementary FE model depicted in Fig. $1 \mathrm{~b}$ Note that $R_{D C}$ stands for the winding DC resistance.

\section{TIME-Domain EXTENSION}

\section{A. Foster Network Approach}

The frequency-dependent equivalent reluctivity $\boldsymbol{\nu}_{e}$ and impedance $\boldsymbol{Z}_{e}$ can be represented over the frequency range of interest by rational functions with a classical Debye model [20]. Therefore, the $m$-order approximation of (6) and (7) are:

$$
\begin{gathered}
\boldsymbol{\nu}_{e}(\boldsymbol{s}) \simeq \nu_{0}+\boldsymbol{s}\left(\ell+\sum_{i=1}^{m} \frac{k_{i}}{1+s g_{i}}\right), \\
\boldsymbol{Z}_{e}(\boldsymbol{s}) \simeq R_{D C}+\boldsymbol{s}\left(L+\sum_{i=1}^{m} \frac{K_{i}}{1+s G_{i}}\right),
\end{gathered}
$$

where $s=\imath \omega$. By restricting the Laplace variable $s$ to be purely imaginary, the frequency-domain response is obtained [16], [20]. The parameters $\ell, L, k_{i}, K_{i}, g_{i}$ and $G_{i}$ can be

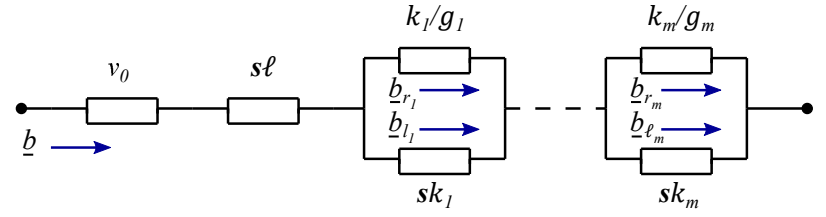

(a)

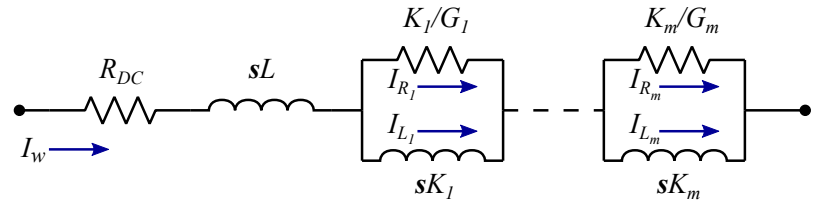

(b)

Fig. 4. Equivalent Foster networks: (a) magnetic and (b) electric.

obtained using the well-known Vector-Fitting technique [15]. The choice of $m$ depends on the treated elementary cell and the frequency band of operation. In this case, $m=2$ provides accurate results as shown in Figs. 2 and 3 , where the fitted curves for $\nu_{e}\left(\nu_{e}^{r}\right.$ and $\left.\nu_{e}^{i} \mathrm{VF}\right)$ and $\boldsymbol{Z}_{e}\left(R_{e}\right.$ and $\left.X_{e} \mathrm{VF}\right)$ are depicted.

Equations (8) and (9) can be straightforwardly associated to an equivalent Foster network with passive elements. The equivalent magnetic network in Fig. $4 \mathrm{a}$ accounts for the proximity effect, whereas the equivalent electric network in Fig. $4 \mathrm{~b}$ accounts for the skin effect. It is worth mentioning that the Debye model satisfies the Kramers-Kronig relation in the frequency domain; thus, causality is guaranteed in the time domain [20].

\section{B. Time-Domain Formulation}

In the winding subdomain $\Omega_{w}$, the time-domain weak formulation is obtained from (1) and (3), including (8) and (9), via the inverse Laplace transform, i.e.

$$
\begin{aligned}
& \nu_{e} *\left[M_{w}\right]\left[A_{w}\right]-\left[C_{w}^{\prime}\right] I_{w}=0, \\
& {\left[C_{w}^{\prime}\right]^{\top} \partial_{t}\left[A_{w}\right]+Z_{e} * I_{w}=V_{w},}
\end{aligned}
$$

where

$$
\begin{array}{r}
\nu_{e}=\nu_{0} \delta+\ell \partial_{t} \delta+\sum_{i=1}^{m} \frac{k_{i}}{g_{i}}\left(\delta-\frac{\mathrm{e}^{-t / g_{i}}}{g_{i}}\right), \\
Z_{e}=R_{D C} \delta+L \partial_{t} \delta+\sum_{i=1}^{m} \frac{K_{i}}{G_{i}}\left(\delta-\frac{\mathrm{e}^{-t / G_{i}}}{G_{i}}\right),
\end{array}
$$

$\delta$ is the Dirac delta function, $\left[M_{w}\right] \in \mathbb{R}^{N_{w} \times N_{w}}$ the winding reluctivity-independent stiffness matrix (with $\nu_{e} *\left[M_{w}\right] \subset$ $[M]$ ), $\left[A_{w}\right] \in \mathbb{R}^{N_{w} \times 1}$ the winding MVP unknown vector (with $\left.\left[A_{w}\right] \subset[A]\right),\left[C_{w}^{\prime}\right] \in \mathbb{R}^{N_{w} \times 1}$ the nonzero part of $\left[C_{w}\right]$ and the $*$ denotes a convolution. By performing the convolution product, the $i$-th terms of 12 and 13 can be expressed as

$$
\begin{gathered}
\left(\nu_{0}+\frac{k_{i}}{g_{i}}\right)\left[M_{w}\right]\left[A_{w}\right]+\ell\left[M_{w}\right] \partial_{t}\left[A_{w}\right]-\left[J_{r_{i}}\right]-\left[C_{w}^{\prime}\right] I_{w}=0, \\
{\left[C_{w}^{\prime}\right]^{\top} \partial_{t}\left[A_{w}\right]+\left(R_{D C}+\frac{K_{i}}{G_{i}}\right) I_{w}+L \partial_{t} I_{w}-V_{r_{i}}=V_{w},}
\end{gathered}
$$


where

$$
\begin{gathered}
{\left[J_{r_{i}}(t)\right]=\left[M_{w}\right] \int_{0}^{t} \frac{k_{i}}{g_{i}^{2}} \mathrm{e}^{-\tau / g_{i}}\left[A_{w}(t-\tau)\right] d \tau} \\
V_{r_{i}}(t)=\int_{0}^{t} \frac{K_{i}}{G_{i}^{2}} \mathrm{e}^{-\tau / G_{i}} I_{w}(t-\tau) d \tau
\end{gathered}
$$

The term $\left[J_{r}\right] \in \mathbb{R}^{N_{w} \times 1}$ can be considered as a reaction current density that includes the dynamic response of the parallel branches in Fig. 4a. Likewise, $V_{r}$ is the reaction voltage that accounts for the dynamic response of the parallel branches in Fig. $4 b$

In (16) and (17), the exponential functions allow a simple recursive evaluation of the convolutions [16], [21]. To that end, let $\Delta t$ be a time step defining: $t_{\mathrm{n}}=t_{\mathrm{n}-1}+\Delta t$, so that, for instance, the recursive form of $[16)$, relying on the properties of the exponential, is given by

$$
\begin{array}{r}
{\left[J_{r_{i}}\left(t_{\mathrm{n}}\right)\right]=\left[M_{w}\right] \int_{0}^{\Delta t} \frac{k_{i}}{g_{i}^{2}} \mathrm{e}^{-\tau / g_{i}}\left[A_{w}\left(t_{\mathrm{n}}-\tau\right)\right] d \tau} \\
+\mathrm{e}^{-\Delta t / g_{i}}\left[J_{r_{i}}\left(t_{\mathrm{n}-1}\right)\right] .
\end{array}
$$

In that way, $\left[J_{r_{i}}\left(t_{\mathrm{n}}\right)\right]$ can be exactly expressed as a function of the previous value $\left[J_{r_{i}}\left(t_{\mathrm{n}-1}\right)\right]$. A complete discrete representation is then obtained through a piece-wise linear approximation of the integral in the right-hand side of (18), i.e.

$$
\begin{array}{r}
{\left[J_{r_{i}}\left(t_{\mathrm{n}}\right)\right]=\theta_{i}\left[J_{r_{i}}\left(t_{\mathrm{n}-1}\right)\right]+v_{i}\left[M_{w}\right]\left[A_{w}\left(t_{\mathrm{n}}\right)\right]} \\
+\psi_{i}\left[M_{w}\right]\left[A_{w}\left(t_{\mathrm{n}-1}\right)\right]
\end{array}
$$

where

$$
\begin{gathered}
\theta_{i}=\mathrm{e}^{-\Delta t / g_{i}}, \\
v_{i}=\frac{k_{i}}{g_{i}}\left(1-\frac{1-\theta_{i}}{1 / g_{i} \Delta t}\right), \\
\psi_{i}=\frac{k_{i}}{g_{i}}\left(\frac{1-\theta_{i}}{1 / g_{i} \Delta t}-\theta_{i}\right) .
\end{gathered}
$$

Analogously, the recursive form of the convolution in 17 is given by

$$
V_{r_{i}}\left(t_{\mathrm{n}}\right)=\Theta_{i} V_{r_{i}}\left(t_{\mathrm{n}-1}\right)+\Upsilon_{i} I_{c}\left(t_{\mathrm{n}}\right)+\Psi_{i} I_{w}\left(t_{\mathrm{n}-1}\right),
$$

where $\Theta_{i}, \Upsilon_{i}$ and $\Psi_{i}$ are obtained as in 20, 22, with the respective parameters $K_{i}$ and $G_{i}$.

\section{Loss and Magnetic Energy}

In the time domain, the equivalent networks in Fig. 4 are preserved, which allows the computation of the losses and the magnetic energy in the post-processing stage. The advantage consists in the possibility to separate the contributions due to the skin and proximity effects. The proximity- and skin-effect losses, $P_{p e}$ and $P_{s e}$, are computed from the flux densities $\underline{b}_{l_{i}}$ and the currents $I_{R_{i}}$ as

$$
\begin{gathered}
P_{p e}=\int_{\Omega_{w}}\left(\partial_{t} \underline{b}^{2} \ell+\sum_{i=1}^{m} \partial_{t} \underline{b}_{\ell_{i}}^{2} k_{i}\right) d \Omega_{w}, \\
P_{s e}=I_{w}^{2} R_{D C}+\sum_{i=1}^{m} I_{R_{i}}^{2} \frac{K_{i}}{G_{i}} .
\end{gathered}
$$

Analogously, the proximity- and skin-effect contributions to the magnetic energy, $W_{p e}$ and $W_{s e}$, are computed from the flux densities $\underline{b}_{r_{i}}$ and the currents $I_{L_{i}}$, i.e.

$$
\begin{gathered}
W_{p e}=\frac{1}{2} \int_{\Omega_{w}}\left(\underline{b}^{2} \nu_{0}+\sum_{i=1}^{m} \underline{b}_{r_{i}}^{2} \frac{k_{i}}{g_{i}}\right) d \Omega_{w}, \\
W_{s e}=\frac{1}{2}\left(I_{w}^{2} L+\sum_{i=1}^{m} I_{L_{i}}^{2} K_{i}\right) .
\end{gathered}
$$

It is worth mentioning that no additional unknowns are required in the FE resolution stage, since $I_{R_{i}}, I_{L_{i}}, \underline{b}_{\ell_{i}}$ and $\underline{b}_{r_{i}}$ are obtained directly from $I_{w}, \underline{b}$ and the circuit parameters.

\section{Computational Cost}

The convolutions (19) and (23) must be calculated at each time step: whereas $V_{r}$ is mesh independent, $\left[J_{r}\right]$ varies with $N_{w}$. The computational cost of $\left[J_{r}\right]$ depends therefore on the mesh size. Moreover, the formulation in (12) and 13 is defined for a scalar reluctivity. An extra convolution in the form of (19) is required, if a tensorial reluctivity is considered. However, it should be noted that the approximation order $m$ has little effect on the computational cost since it does not add degrees of freedom to the resolution stage. Thus, on account of the convolutions, the proposed approach requires a small extra computational cost compared to the traditional stranded model (see Section IV).

\section{APPliCATION}

\section{A. Model Performance}

The proposed homogenized approach is first applied to a planar 2-D FE model of the 256-turn inductor shown in Fig. 5 with the conductor characterized in Section II. Only 1/4th of the domain is considered in Fig. 5 by taking advantage of symmetry. Thus, tangential magnetic field is imposed on the left boundary and normal magnetic field on the bottom boundary. Moreover, a magnetic shielding box covers the core and winding (normal magnetic flux boundary condition on its inner surface). The core is considered nonconductive and has relative reluctivity $\nu_{r}=1 / 1000$. The winding is fed by the $2 \mathrm{kHz}$ PWM signal, of unit amplitude and $50 \%$ duty cycle, shown in Fig 6. Time-stepping simulations are carried out for one period $(T=0.5 \mathrm{~ms})$ with time step $\Delta t=T / 500$. The results are compared to those obtained with: a reference FE model (in which all turns explicitly defined as solid conductors) and the traditional stranded model. The computations of the reference and stranded models are carried out with the software Altair Flux ${ }^{\mathrm{TM}}$ [23], whereas the proposed homogenized approach is developed in the MATLAB environment.

The mesh of the reference case is presented in Fig. 7a where its fine discretization inside the conductors accurately accounts for the eddy-current effects. This fine mesh leads to a total of 77965 unknowns, comprising the whole domain of Fig. 5. As for the homogenized and stranded cases, the discretization of the winding region results in the much coarser mesh shown in Fig. 7b, which contains 1466 unknowns, 


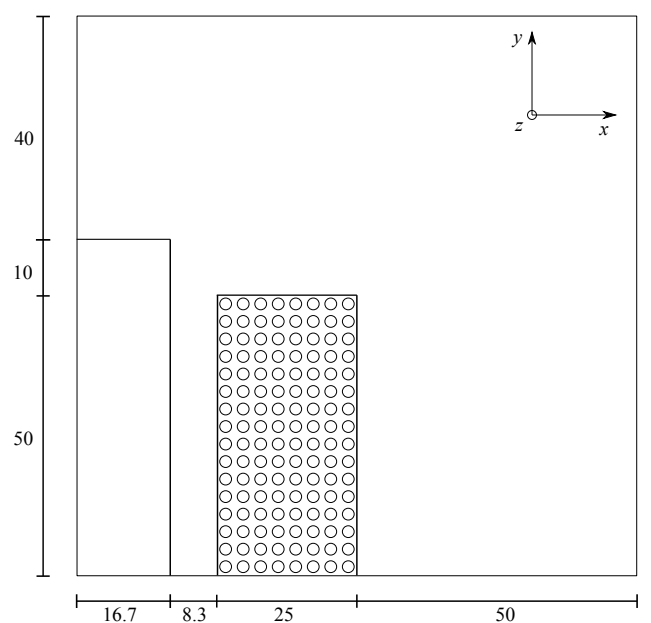

Fig. 5. 2-D inductor (1/4th upper-half, depth: $0.5 \mathrm{~m}$, dimensions: $\mathrm{mm}$ ).

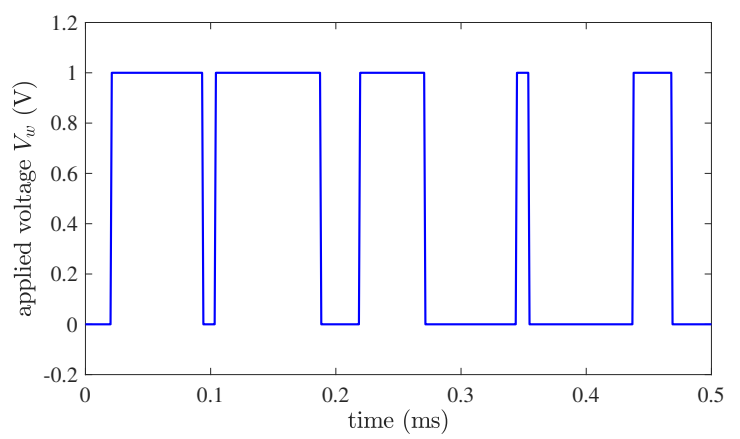

Fig. 6. PWM signal of the imposed voltage.

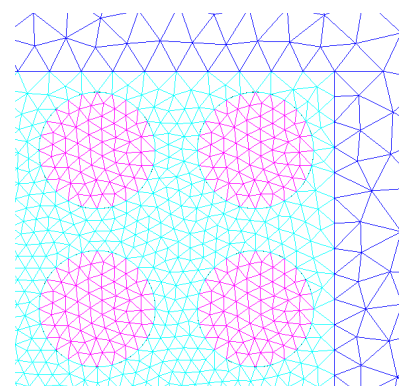

(a)

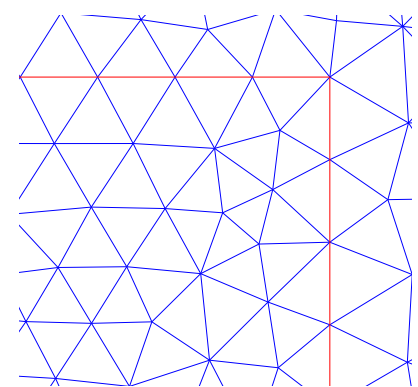

(b)
Fig. 7. Model mesh (upper right zoom of the outer turns): (a) reference case and (b) homogenized and stranded cases.

comprising the whole domain of Fig. 5. At the resolution stage, the computational times per step are: $4650 \mathrm{~ms}, 11.4 \mathrm{~ms}$ and $10.4 \mathrm{~ms}$ for the reference, homogenized and stranded models, respectively. The proposed approach delivers a speed-up factor of 400 compared to the reference time. The computational cost of the RC produces a $10 \%$ time increase ( $1 \mathrm{~ms}$ per time step) compared to the stranded model. If the approximation order is doubled to $m=4$, the computational time per step is $11.7 \mathrm{~ms}$ (2.6\% increase), which confirms the little contribution of $m$ to the computational cost.

The flux lines in the winding are shown in Fig. 8 at $t=T / 2$. It can be observed that the global pattern of the flux lines is preserved in the homogenized case. Fig. 9

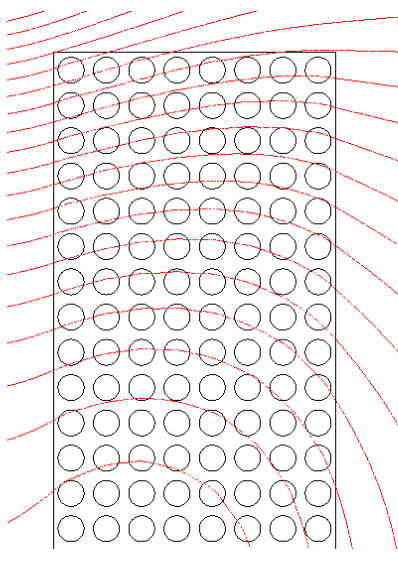

(a)

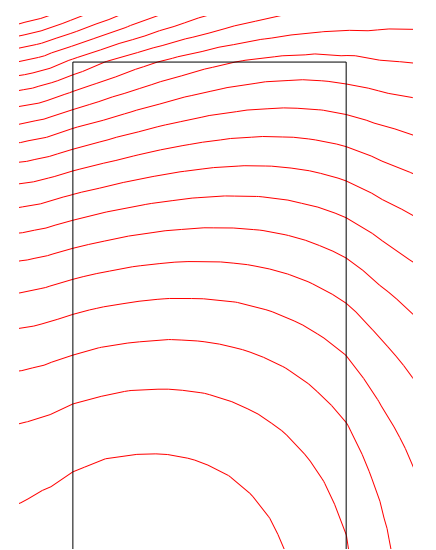

(b)
Fig. 8. Flux lines in the winding at $t=T / 2$ (a) reference case (b) homogenized case.

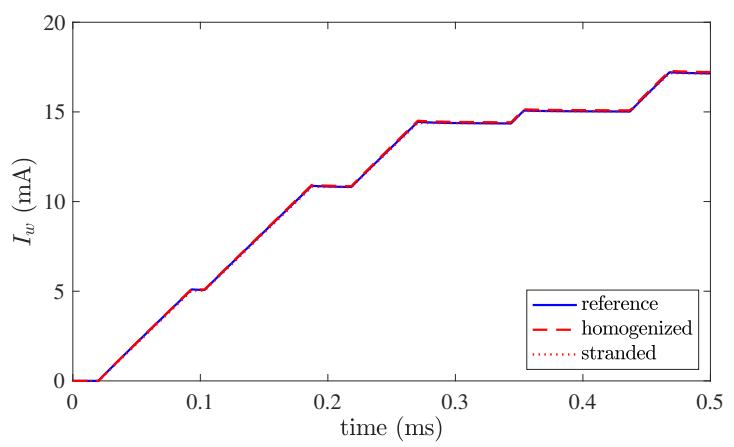

Fig. 9. Current $I_{w}$ versus time obtained with the reference, homogenized and stranded models.

compares the reference, homogenized and stranded winding current variation in time, where both the homogenized and stranded models produce an excellent agreement. The current $I_{w}$ is only influenced by the skin effect, which in this case is negligible; hence no difference can be appreciated between the homogenized and stranded models.

The main objective of the proposed homogenized approach is to accurately account for the Joule losses in the time domain. Fig. 10 shows the Joule losses in time for the reference, homogenized and stranded model. The homogenized model produces an excellent agreement compared to the reference. The stranded model loses accuracy as it only accounts for the DC losses. Following (24) and (25), it is possible to separate the skin and proximity effect contribution to the total losses. The variation in time of the skin and proximity effect losses is also presented in Fig. 10. It should also be noted that the skin-effect losses match the losses of the stranded model since the equivalent impedance $Z_{e}$ is basically describing its DC value. The magnetic energy as a function of time for the reference, homogenized and stranded model, between $0.15 \mathrm{~ms}$ and $0.3 \mathrm{~ms}$, is shown in Fig. 11, the proposed homogenization delivers more accurate results compared to the stranded model.

In order to measure the influence of the homogenized mesh, two additional discretizations are tested: one with a mesh of approximately one third of the mesh elements in Fig. $7 \mathrm{~b}$ and 


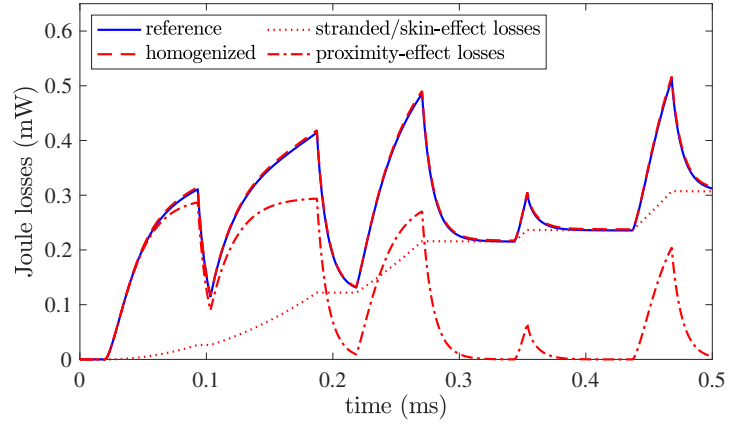

Fig. 10. Joule losses versus time obtained with the reference, homogenized and stranded models.

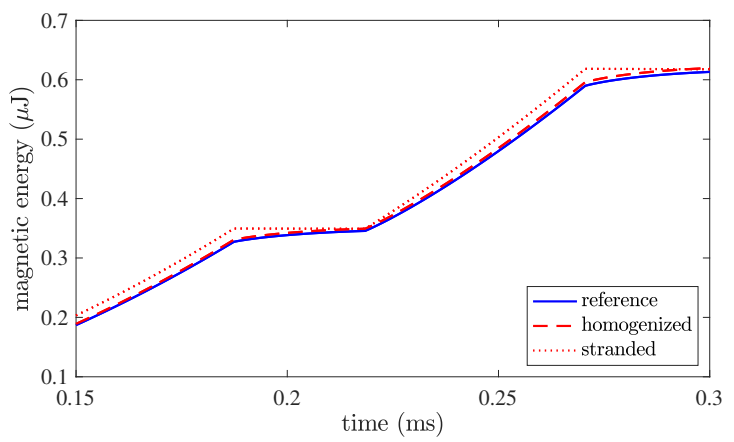

Fig. 11. Magnetic energy versus time obtained with the reference, homogenized and stranded models.

the other with three times the number of elements in Fig. $7 \mathrm{~b}$ The respective computational times per step are $3.39 \mathrm{~ms}$ and $38.3 \mathrm{~ms}$. The results for the Joule losses between $0.1 \mathrm{~ms}$ and $0.2 \mathrm{~ms}$ are presented in Fig. 12. It can be appreciated that the size of the homogenized mesh has little influence on the results.

In terms of accuracy, the proposed homogenization relies on the quality of the Vector-Fitting process [15]. However, an excellent agreement might be achieved with a rather low order of approximation, since the frequency-dependent curves describing the equivalent properties (reluctivity and impendace) are always smooth as in Figs. 2 and 3 [7], [13], [14]. Regarding the adopted RC scheme, it has been proved that the scheme is convergent independently of the time-step size; even if the accuracy of the solution depends on the selection of the time step, a matching solution by RC has always been obtained [21]. Moreover, the RC scheme avoids truncation in the integration due to the mathematical redefinition in (18) [21].

A secondary winding can be considered, in the case of transformer modeling, as long as it belongs to a separate region with and independent circuit relation following the considerations in Section II [24]. It is worth noting that the elementary cell may vary from winding to winding. Conductive nonlinear materials, proper to ferromagnetic cores, can be straightforwardly considered in the winding surroundings. Furthermore, a post-processing could be performed to estimate the local current density and eddy-current losses that would serve as boundary conditions in a thermal problem. This way, the temperature distribution could be estimated [25].

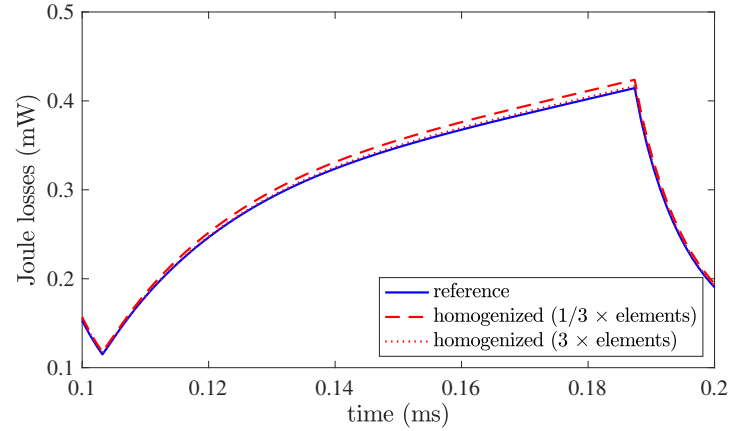

Fig. 12. Homogenized Joule losses versus time obtained with $1 / 3$ and 3 times the mesh elements in Fig. $7 \mathrm{~b}$

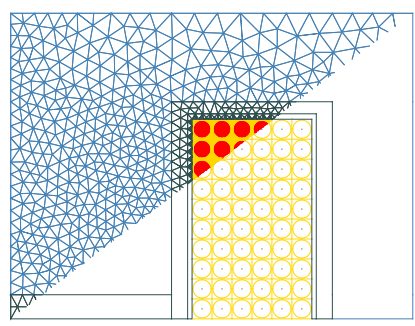

(a)

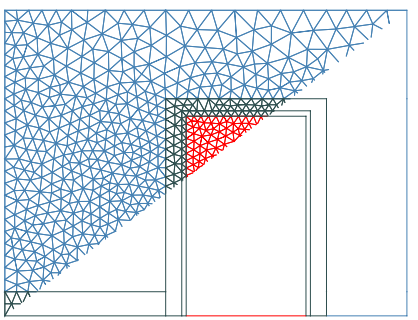

(b)
Fig. 13. Geometry and meshes of the axisymmetric inductor (a) reference case and (b) homogenized case.

\section{B. Comparison with an RL Cauer Approach}

A second test case is considered to compare the proposed approach to the one in [13], [14], where the frequencydependent parameters are approximated with RL Cauer networks instead (see Appendix A). To that end, we consider the 120-turn axisymmetric inductor shown in Fig. 13 with the conductor characterized in [14] (a round conductor with square packing (radius $r=0.56 \mathrm{~mm}, \sigma=59 \mathrm{MS} / \mathrm{m}$ and $\lambda=0.65$ ). The core is considered nonconductive and has relative magnetic reluctivity $\nu_{r}=1 / 1000$. Tangential magnetic field is imposed on the outer boundary of the core. The winding is fed by a $50 \mathrm{kHz}$ sinusoidal voltage of peak amplitude $25 \mathrm{~V}$. Time-stepping simulations are carried out for one period with time step $\Delta t=T / 120$. The homogenized approaches are additionally compared to a reference FE model (with all turns explicitly defined as solid conductors) whose results can be obtained with the software Altair Flux ${ }^{\mathrm{TM}}[23]$. In this subsection, we refer to the proposed homogenized approach and the one in [13], [14] as Foster and Cauer, respectively. A detail of the meshes for the reference and homogenized cases are presented in Fig. 13a and 13b respectively. The fine mesh in Fig. 13a leads to a total of 12534 unknowns with a computational time per step of $375 \mathrm{~ms}$.

We focus our comparison on the Joule losses, since they are highly sensitive to the eddy-current effects. It is of interest to estimate the accuracy of the approaches as the degree of approximation $m$ increases. Thus, we vary the approximation order $m$ from 1 to 4 and compute the Joule losses for each order. In Fig. 14 such computations are presented and compared to the reference case, where the "C." and the "F." 


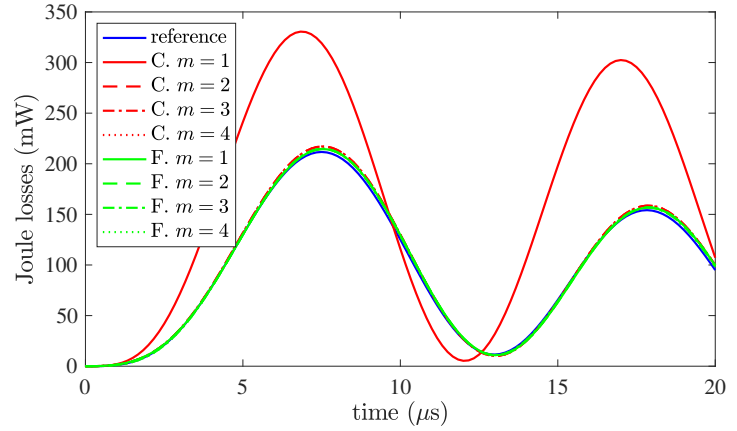

Fig. 14. Joule losses versus time obtained with the reference and homogenized approaches.

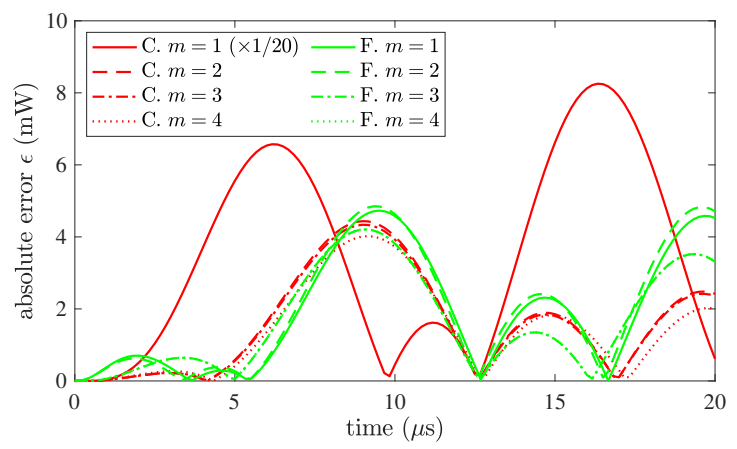

Fig. 15. Absolute error $\epsilon$ versus time for the Joule losses obtained with the homogenized approaches.

TABLE I

PERFORMANCE OF THE HOMOGENIZED MODELS

\begin{tabular}{ccccccc}
\hline \hline \multirow{3}{*}{$m$} & \multicolumn{3}{c}{ RL Cauer } & \multicolumn{3}{c}{ RL Foster } \\
& $\tilde{N}_{w}$ & $t_{\Delta t}(\mathrm{~ms})$ & $\epsilon_{L 2}(\%)$ & $N_{w}$ & $t_{\Delta t}(\mathrm{~ms})$ & $\epsilon_{L 2}(\%)$ \\
\hline 1 & 500 & 38 & 75 & 500 & 5 & 2.1 \\
2 & 1000 & 91 & 2.8 & 500 & 5 & 2.1 \\
3 & 1500 & 129 & 1.8 & 500 & 5 & 1.8 \\
4 & 2000 & 165 & 1.6 & 500 & 5 & 1.8 \\
\hline \hline
\end{tabular}

stand for the Cauer and Foster approaches, respectively. The biggest difference concerns the first-order approximation: the Cauer approach is incapable of following the behavior of the reference, whereas the Foster approach has very good accuracy. As from $m=2$, both approaches deliver excellent results. For a better insight, Fig. 15 shows the absolute error $\epsilon$ in terms of the Joule losses for both methods. On one hand, a considerable improvement is observed for the Cauer approach from $m=1$ to $m=2$. If the approximation increases further, slight improvements in the accuracy are also obtained. On the other hand, the Foster approach produces an excellent stable accuracy at all orders of approximation, but again with a small gain at higher orders.

Table I summarizes the performance of both models comparing the unknowns in the winding region $N_{w}$, the computational time per step $t_{\Delta t}$ and the L2-error: $\epsilon_{L 2}=\| P_{r}-$ $P_{h}\left\|_{2} /\right\| P_{r} \|_{2}$, with $P_{r}$ the vector of reference values and $P_{h}$ the vector of homogenized values. The Cauer approach delivers the lowest error at the expense of a high computational cost resulting from the additional unknowns in $\Omega_{w}$. The Foster approach on the contrary performs with the same computational cost regardless the value of $m$, since it is not linked to the unknowns of the FE problem. At the highest order, a difference of only $0.2 \%$ in $\epsilon_{L 2}$ is found between the approaches.

\section{CONCLUSIONS}

An alternative approach has been proposed for the timedomain homogenization of multiturn windings. It allows to solve the FE model, including the eddy-current effects, with excellent accuracy and reasonable computational cost. To this end, the winding type is first characterized by a 2-D elementary model, leading to frequency-dependent equivalent parameters. These parameters are associated to an equivalent Foster network and translated into the time domain via the inverse Laplace transform. The time-dependent formulation is then solved with a RC scheme. No additional unknowns need to be introduced in the model, which reduces the computational cost. In comparison with an homogenization in which the parameters are associated to an equivalent Cauer network, the proposed Foster approach: performs better at the lowest order of approximation and has a computational cost independent of the approximation order.

\section{APPENDIX A}

\section{RL CAUER NETWORK APPROACH}

The complex frequency-dependent parameters $\boldsymbol{Z}_{e}$ and $\boldsymbol{\nu}_{e}$ can be represented, over the frequency range of interest, by rational functions with a ladder form, i.e.

$$
\begin{aligned}
& \boldsymbol{Z}_{e}(\boldsymbol{s}) \simeq R_{1}+\frac{1}{\frac{1}{s L_{1}}+\frac{1}{R_{2}+\frac{1}{\frac{1}{s L_{2}}+\ddots}}}, \\
& \boldsymbol{\nu}_{e}(\boldsymbol{s}) \simeq \mathfrak{R}_{1}+\frac{1}{\frac{1}{\boldsymbol{s} \mathfrak{L}_{1}}+\frac{1}{\mathfrak{R}_{2}+\frac{1}{\frac{1}{s \mathfrak{L}_{2}}+\ddots}}},
\end{aligned}
$$

where the fractions can be expanded up to an $m$-order. The parameters $R_{1}, L_{1}, \mathfrak{R}_{1}, \mathfrak{L}_{1}, \ldots$ are obtained through the fitting of the admittance $\boldsymbol{Y}_{e}(\boldsymbol{s})=1 / \boldsymbol{Z}_{e}(\boldsymbol{s})$ and permeability $\boldsymbol{\mu}_{e}(\boldsymbol{s})=1 / \boldsymbol{\nu}_{e}(\boldsymbol{s})$ that match rational, causal and stable transfer functions [14].

The behavior of (28) and (29) can be represented by RL Cauer networks in the frequency-domain. In the time domain, the RL network constitutes a linear and time invariant (LTI) system of order $m$ [13]. For the skin effect, an approximate dynamic relation between the instantaneous terminal voltage $V_{w}$ and the current $I_{w}$ considers $m-1$ auxiliary currents and a system of $m$ first-order differential equations in terms of the $m$ currents $\left[I_{w}\right]^{\top}=\left[\begin{array}{llll}I_{w} & I_{2} & I_{3} \ldots\end{array}\right]^{\top}$. Likewise, a dynamic relation between the instantaneous magnetic field $\underline{h}$ and the magnetic flux density $\underline{b}$ can be obtained by considering $m-1$ auxiliary 
flux densities and a system of $m$ first-order differential equations in terms of the $m$ flux densities $[B]^{\top}=\left[\underline{b}_{2} \underline{b}_{2} \underline{b}_{3} \ldots\right]^{\top}$. The relations read:

$$
\begin{aligned}
{\left[V_{w}\right] } & =[R]\left[I_{w}\right]+[L] \partial_{t}\left[I_{w}\right], \\
{[H] } & =[\mathfrak{R}][B]+[\mathfrak{L}] \partial_{t}[B],
\end{aligned}
$$

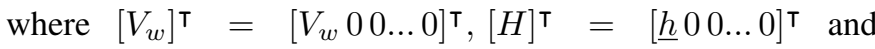
$[R, L, \mathfrak{R}, \mathfrak{L}] \in \mathbb{R}^{m \times m}$ are the constant symmetric matrices associated to the RL networks [14].

In the winding domain $\Omega_{w}$, the auxiliary flux densities are considered through auxiliary components of the MVP with the same basis functions, where their nodal values constitute ad-

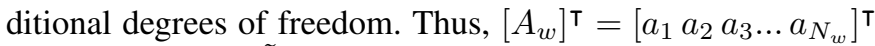
is redefined as $\left[\tilde{A}_{w}\right]^{\top}=\left[\begin{array}{lllll}a_{1,1} & a_{2,1} & a_{3,1} & \ldots & a_{m, N_{w}}\end{array}\right]^{\top}$, so that $\left[\tilde{A}_{w}\right] \in \mathbb{R}^{\tilde{N}_{w} \times 1}$ with $\tilde{N}_{w}=N_{w} m[13]$. Therefore, the timedomain weak formulation, obtained from (1) and (3) and including (30) and (31), in $\Omega_{w}$ reads:

$$
\begin{gathered}
{\left[\tilde{M}_{w}\right][\Re]\left[\tilde{A}_{w}\right]+\left[\tilde{M}_{w}\right][\mathfrak{L}] \partial_{t}\left[\tilde{A}_{w}\right]-\left[\tilde{C}_{w}^{\prime}\right]\left[I_{w}\right]=0} \\
{\left[\tilde{C}_{w}^{\prime}\right]^{\top} \partial_{t}\left[\tilde{A}_{w}\right]+[R]\left[I_{w}\right]+[L] \partial_{t}\left[I_{w}\right]=\left[V_{w}\right]}
\end{gathered}
$$

where $\left[\tilde{M}_{w}\right] \in \mathbb{R}^{\tilde{N}_{w} \times \tilde{N}_{w}}$ and $\left[\tilde{C}_{w}^{\prime}\right] \in \mathbb{R}^{\tilde{N}_{w} \times 1}$. The formulation in (32) and (33) is defined for a scalar reluctivity. An extra equivalent parameter in the form of (29) is needed if a tensorial reluctivity is considered e.g., elementary cells with $l_{x} \neq l_{y}$.

\section{REFERENCES}

[1] I.T. Gürbüz, A. Lehikoinen, U. Aydin, P. Rasilo, E. Takala and A Belahcen, " Domain decomposition with subdomain pre-processing for finite element modelling of transformers with stranded conductors," in AIP Advances, vol. 10, no. 5, 2020.

[2] A. Marjamäki and P. Rasilo, "Domain decomposition technique with subdomain pre-processing in 2-D simulations of wireless power transfer," in IEEE Trans. Magn., vol. 56, no. 4, pp. 1-4, April 2020.

[3] L. Lehti, J. Keränen, S. Suuriniemi and L. Kettunen, "Coil winding losses: decomposition strategy," in IEEE Trans. Magn., vol. 52, no. 1, pp. 1-6, Jan. 2016.

[4] R. V. Sabariego, C. Geuzaine, P. Dular and J. Gyselinck, "Time-domain surface impedance boundary conditions enhanced by coarse volume finiteelement Discretisation," in IEEE Trans. Magn., vol. 48, no. 2, pp. 631634, Feb. 2012

[5] S. Yuferev and L. Di Rienzo, "Surface impedance boundary conditions in terms of various formalisms," in IEEE Trans. Magn., vol. 46, no. 9, pp. 3617-3628, Sept. 2010.

[6] C. Golovanov, Y. Marechal and G. Meunier, "3D edge element based formulation coupled to electric circuits," in IEEE Trans. Magn., vol. 34 no. 5, pp. 3162-3165, Sept. 1998.

[7] O. Moreau, L. Popiel and J. L. Pages, "Proximity losses computation with a 2D complex permeability modelling," in IEEE Trans. Magn., vol. 34, no. 5, pp. 3616-3619, Sept. 1998.

[8] H. Igarashi, "Semi-analytical approach for finite-element analysis of multi-turn coil considering skin and proximity effects," IEEE Trans. Magn., vol. 53, no. 1, pp. 1-7, Jan. 2017.

[9] D. C. Meeker, "An improved continuum skin and proximity effect model for hexagonally packed wires," in Journal of Computational and Applied Mathematics, vol. 236, no. 18, pp. 4635-4644, 2012.

[10] G. Meunier, V. Charmoille, C. Guerin, P. Labie and Y. Marechal, "Homogenization for periodical electromagnetic structure: which formulation?," in IEEE Trans. Magn., vol. 46, no. 8, pp. 3409-3412, Aug. 2010.

[11] J. Gyselinck and P. Dular, "Frequency-domain homogenization of bundles of wires in 2-D magnetodynamic FE calculations," in IEEE Trans. Magn., vol. 41, no. 5, pp. 1416-1419, May 2005.

[12] A. Phung, G. Meunier, O. Chadebec, X. Margueron and J. Keradec, "High-frequency proximity losses determination for rectangular crosssection conductors," in IEEE Trans. Magn., vol. 43, no. 4, pp. 1213-1216, Apr. 2007.
[13] J. Gyselinck, R. V. Sabariego and P. Dular, "Time-domain homogenization of windings in 2-D finite element models," in IEEE Trans. Magn., vol. 43, no. 4, pp. 1297-1300, April 2007.

[14] Niyomsatian K, Gyselinck J, Sabariego RV. Time-domain homogenization of multiturn windings based on RL Cauer ladder networks. Int J Numer Model. 2019; 2649.

[15] B. Gustavsen and A. Semlyen, "Rational approximation of frequency domain responses by vector fitting," in IEEE Trans. Power Del., vol. 14, no. 3, pp. 1052-1061, July 1999.

[16] S. Grivet-Talocia, B. Gustavsen, Passive Macromodeling. Hoboken, New Jersey: John Wiley \& Sons, Inc., 2016, p. 16.

[17] L. Liu, X. Cui and L. Qi, "Simulation of electromagnetic transients of the bus bar in substation by the time-domain finite-element method," in IEEE Trans. Electromagn. Compat., vol. 51, no. 4, pp. 1017-1025, Nov. 2009.

[18] M. Tang and J. Mao, "A precise time-step integration method for transient analysis of lossy nonuniform transmission lines," in IEEE Trans. Electromagn. Compat., vol. 50, no. 1, pp. 166-174, Feb. 2008.

[19] K. M. Coperich, J. Morsey, V. I. Okhmatovski, A. C. Cangellaris and A. E. Ruehli, "Systematic development of transmission-line models for interconnects with frequency-dependent losses," in IEEE Trans. Microw. Theory Tech., vol. 49, no. 10, pp. 1677-1685, Oct. 2001.

[20] M. S. Sarto, A. Scarlatti and C. L. Holloway, "On the use of fitting models for the time-domain analysis of problems with frequency-dependent parameters," IEEE EMC International Symposium. Symposium Record., vol.1 pp. 588-593, 2001.

[21] A. Semlyen and A. Dabuleanu, "Fast and accurate switching transient calculations on transmission lines with ground return using recursive convolutions," in IEEE Trans. Power App. Syst., vol. 94, no. 2, pp. 561571, 1975.

[22] P. Dular, F. Henrotte and W. Legros, "A general and natural method to define circuit relations associated with magnetic vector potential formulations," in IEEE Trans. Magn., vol. 35, no. 3, pp. 1630-1633, 1999.

[23] Altair Flux ${ }^{T M}$. (2019.1), Altair Engineering. Accessed: Feb. 20, 2020. [Online]. Available: https://www.altair.com/flux/

[24] K. Niyomsatian, J. Gyselinck and R. V. Sabariego, "Closed-form complex permeability expression for proximity-effect homogenisation of litzwire windings," in IET Science, Measurement \& Technology, vol. 14, no. 3, pp. 287-291, 52020.

[25] L. Idoughi, X. Mininger, F. Bouillault, L. Bernard and E. Hoang, "Thermal model with winding homogenization and FIT discretization for stator slot," in IEEE Trans. Magn., vol. 47, no. 12, pp. 4822-4826, 2011.

[26] J. Gyselinck, P. Dular, C. Geuzaine and R. V Sabariego, "Direct inclusion of proximity-effect losses in two-dimensional time-domain finite-element simulation of electrical machines," Proceedings of the 8th International Symposium on Electric and Magnetic Fields, Mondovi, Italy, 2009. 